\title{
Resultados clínicos del tratamiento de frenillos labiales; frenectomía y frenotomía.
}

\section{Clinical results of the treatment of labial frenulums; frenectomy and frenotomy.}

\author{
Yuri Castro-Rodríguez ${ }^{1 *}$, Francis Bravo-Castagnola1 ${ }^{1}$, Sixto Grados-Pomarino
}

\author{
1. Especialidad de Periodoncia e Implantología. \\ Universidad Nacional Mayor de San Marcos. Lima. \\ Perú. \\ * Correspondencia Autor: Yuri Castro R. | Jr. Tomás \\ Catari 463, Urb. El Trébol. Dpto. 201. Los Olivos, \\ Perú. | Celular: 989836354 | e-mail: yuricastro_16@ \\ hotmail.com \\ Trabajo recibido el 02/02/2017. \\ Aprobado para su publicación el 22/06/2017
}

\begin{abstract}
RESUMEN
Objetivo: El propósito de esta revisión fue evaluar sistemáticamente la literatura científica sobre los resultados clínicos que se obtienen al remover los frenillos aberrantes al utilizar los procedimientos quirúrgicos convencionales y el láser. Materiales y métodos: Búsqueda detallada en las bases de datos PubMed, ScienceDirect, Cochrane y SciELO para obtener la información más actualizada los resultados clínicos (dolor posoperatorio, dolor al hablar, dolor al masticar y complicaciones posquirúrgicas) entre las técnicas convencionales (uso del escalpelo) y las técnicas de remoción con el láser (búsqueda de ensayos clínicos). Resultados: De los 501 archivos identificados inicialmente, se excluyeron reportes clínicos, revisiones, estudios observacionales, comentarios, estudios con pacientes pediátricos, etc. Se incluyeron cuatro ensayos clínicos que contrastaron las variables entre la técnica convencional y las técnicas con el láser $\mathrm{Nd}$ : YAG y láser $\mathrm{CO}_{2}$ Conclusión: La percepción del dolor posoperatorio, el dolor al hablar y el disconfort al masticar luego de la frenectomía es menor en las técnicas que utilizan el láser de $\mathrm{Nd}$ : YAG y $\mathrm{CO}_{2}$ frente a las técnicas que utilizan el escalpelo. El tiempo quirúrgico con el uso del láser es significativamente menor que al utilizar el escalpelo. La heterogeneidad de los estudios limita la realización de un metanálisis con respecto a los resultados.
\end{abstract}

PALABRAS CLAVE

Frenectomía; Frenillo labial; Cirugía bucal.

International Journal of Interdisciplinary Dentistry Vol. 13(1) 40-43, 2020.

\begin{abstract}
Objective: The purpose of this review was to evaluate systematically the scientific literature on the clinical results obtained by removing aberrant frenulums using conventional surgical procedures and laser. Materials and methods: A detailed search was performed in the PubMed, ScienceDirect, Cochrane and SciELO databases to obtain the most up-to-date clinical results (postoperative pain, pain when speaking, pain when chewing and postoperative complications) among conventional (scalpel use) and laser removal techniques (search for clinical trials). Results: Of the 501 records initially identified, clinical reports, reviews, observational studies, comments, studies with pediatric patients, etc. were excluded. We included four clinical trials that contrasted the variables between the conventional technique and the techniques with the $\mathrm{Nd}$ : YAG laser and $\mathrm{CO}_{2}$ laser. Conclusion: The perception of postoperative pain, pain when speaking and chewing discomfort after frenectomy is lower in the techniques using the $\mathrm{Nd}$ : YAG and $\mathrm{CO}_{2}$ laser versus the techniques using the scalpel. The surgical time when using of laser is significantly lower than when using the scalpel. The heterogeneity of the studies limits the performance of a meta-analysis with respect to the results. KEY WORDS

Frenectomy; Labial frenum; Oral surgery.
\end{abstract}

International Journal of Interdisciplinary Dentistry Vol. 13(1) 40-43, 2020.

\section{INTRODUCCIÓN}

La inserción de los frenillos labiales raramente ocasiona patologías dentro de la cavidad bucal; sin embargo, cuando lo hace puede ocasionar problemas periodontales, fonéticos, ortodónticos y protésicos ${ }^{(1)}$.

Los frenillos con inserción larga pueden retraer el margen gingival e iniciar la progresión de la enfermedad periodontal; sin embargo, no hay una clara relación de causa-efecto entre la inserción del frenillo y el origen de una recesión gingival(2). Lo que más ha sido demostrado es que su inserción elevada interfiere con el cepillado bucal y por ende aumenta el acúmulo de placa bacteriana a nivel del margen gingival(3).

La frenectomía es la remoción completa del frenillo incluida su adherencia al hueso alveolar; mientras que la frenotomía es la incisión y recolocación de la adherencia del frenillo(4). Ambos procedimientos se 
indican cuando: la inserción del frenillo provoque diastemas; prevención del relapso post tratamiento de ortodoncia, cercanía de la inserción al margen gingival que produzca una recesión gingival o altere la higiene oral y cuando se observe una pobre adherencia gingival o una profundidad vestibular disminuida ${ }^{(2,4)}$.

La frenectomía se realiza a través de técnicas convencionales (técnica clásica, técnica de Miller, etc.) técnicas que combinan el uso de bisturíes y colgajos; las otras técnicas remueven los frenillos a través del láser. La frenectomía con láser remueve el frenillo de una forma más rápida y con muchas más ventajas que las técnicas convencionales. Hasta el momento se han utilizado varios tipos de láseres para este procedimiento. Se reportan usos con el láser de $\mathrm{CO}_{2}$, láser de $\mathrm{Nd}$-YAG, láser de Er-YAG y el diodo láser ${ }^{(5)}$.

Las técnicas con el uso del láser demuestran no necesitar de anestesia local, generar menos dolor, otorgar mejor visibilidad al operar, no necesitar apósitos periodontales, otorgar mejor cicatrización y ocasionar una menor cicatriz(5).

La presente revisión sistemática sintetizó las publicaciones más actualizadas sobre los resultados clínicos de las técnicas convencionales frente a las técnicas con láser en la remoción de los frenillos aberrantes.

\section{MATERIALES Y MÉTODOS}

\section{Estrategia de búsqueda}

Los autores exploraron los artículos de las bases de datos PubMed, ScienceDirect, Cochrane y SciELO desde el año 2007 (enero) hasta la actualidad (noviembre del 2016- últimos diez años). La limitación temporal se realizó para buscar los artículos más actuales y que utilicen las técnicas más comunes. La investigación fue complementada con una búsqueda manual desde las mismas fechas en las revistas: Journal of Periodontology, International Journal of Oral \& Maxillofacial Implants, International Journal of Oral \& Maxillofacial Surgery, Journal of Oral and Maxillofacial Surgery, Journal of Dental Research, Journal of Clinical Periodontology, International Journal of Periodontics \& Restorative Dentistry y European Journal of Oral Implantology. Encontrados los artículos se complementó con una búsqueda de las referencias bibliográficas para detectar publicaciones que no fueron identificadas electrónicamente. La estrategia de búsqueda fue realizada por un examinador (YC).

\section{Términos de búsqueda}

Los siguientes términos de búsqueda (palabras clave) fueron utilizados: "Frenectomy", "Frenotomy", "Surgery", "Labial", "Oral”, "Clinical trial”, todos términos MeSH. La utilización de operadores boléanos incluyó los siguientes algoritmos: Frenectomy OR Frenotomy, ((Frenectomy OR Frenotomy) AND Oral) AND Surgery, (((Frenectomy OR Frenotomy) AND Oral) AND Surgery) AND Labial, ((((Frenectomy OR Frenotomy) AND Oral) AND Surgery) AND Labial) AND Clinical trial. Los términos DeCS utilizados incluyeron: "Frenillo labial", "Cirugía bucal" y "Cirugía maxilofacial". Los algoritmos utilizados con los términos DeCS fueron similares a los utilizados con los términos MeSH.

\section{Criterios de inclusión/exclusión}

Los criterios de inclusión fueron los siguientes:

- Publicaciones en inglés o español.

- Ensayos clínicos que comparen técnicas convencionales y técnicas con láser en la remoción de frenillos aberrantes.

- Ensayos clínicos realizados en pacientes adultos.

- Ensayos clínicos que evaluaron las características de dolor, disconfort y cicatrización posoperatoria.

Los criterios de exclusión fueron los siguientes:

- Estudios preclínicos o estudios piloto.

- Ensayos clínicos realizados en niños, o pacientes menores a 18 años.

- Ensayos clínicos que no describan los resultados posoperatorios de dolor, disconfort y cicatrización.

- Estudios clínicos de los tipos: Reportes de caso, series de caso, estudios retrospectivos y revisiones sistemáticas.

\section{Proceso de selección}

La selección se realizó a través de 2 revisores de forma independiente (Y.C, F.B.) Inicialmente todos los títulos fueron seleccionados para luego eliminar las publicaciones no relevantes, en la primera búsqueda se encontraron 501 archivos entre las cuatro bases de datos seleccionadas. Durante una segunda etapa se utilizaron los filtros de cada base de datos seleccionando las opciones de "búsqueda por tiempo", "búsqueda de ensayos clínicos" y "búsqueda de artículos" principalmente en las bases PubMed y ScienceDirect. Los artículos resultantes fueron analizados a través de sus resúmenes; los dos autores seleccionaron de forma independiente todos los resúmenes. El grado de concordancia Kappa entre los dos autores fue de 0.96 para la concordancia de selección de los resúmenes. Luego se obtuvieron los artículos que cumplían los criterios de inclusión. Luego de esta búsqueda, los artículos relevantes y las referencias bibliográficas fueron analizados para añadir más artículos que cumplan con los criterios. Cualquier desacuerdo entre los dos revisores fue resuelto con una discusión adicional (Figura 1).

\section{Evaluación del riesgo de sesgo}

La calidad de los estudios fue evaluada en base a la los criterios del Centro Cochrane ${ }^{(6)}$ y el protocolo CONSORT ${ }^{(7)}$ según los siguientes protocolos: 1) Generación de secuencias, 2) método de asignación de los grupos, 3) enmascaramiento del examinador, 4) ubicación de datos incompletos, 5) informe de resultados. El grado de sesgo fue categorizado como bajo riesgo si todos los criterios fueron cumplidos, moderado riesgo si solo un criterio faltó y de alto riesgo si dos o más criterios faltaron. El proceso de selección de los artículos se resume en la figura 1.

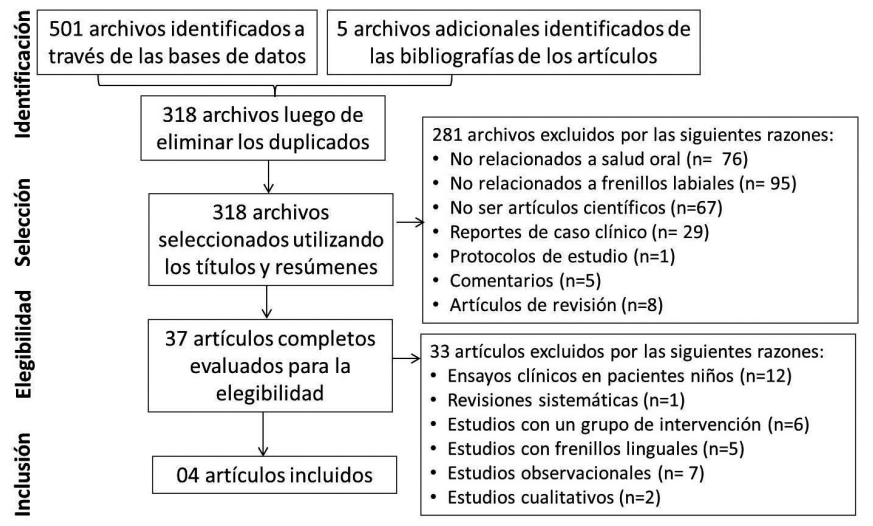

Figura 1. Diagrama de flujo de la revisión sistemática. Cuatro publicaciones incluidas para su análisis

\section{Extracción de datos}

Los datos a extraer incluyeron: 1) técnicas utilizadas, 2) diseño del estudio, 3) tamaño de la muestra, 4) características individuales, 5) evaluación de disconfort, dolor y tiempo quirúrgico, 6) seguimiento diario o semanal. En adición algunos datos sobre complicaciones en la cicatrización de algunos artículos también fueron añadidos.

\section{RESULTADOS}

De las cuatro bases de datos analizadas durante la primera identificación se encontraron 501 archivos relacionados con el tema. Al evaluar la duplicidad de archivos restaron 318; de los cuales 281 fueron excluidos por no estar relacionados a la salud oral, no ser artículos científicos, ser reportes de casos clínicos, etc. (Figura 1). Luego de los procesos de selección y elegibilidad solo cuatro artículos científicos cumplieron con los criterios de inclusión ${ }^{(8-11)}$, centrando la búsqueda y análisis en ensayos clínicos. Tres artículos fueron ensayos clínicos aleatorizados ${ }^{(9-11)}$ y uno fue no aleatorizado(8); sólo uno fue de diseño multicéntrico ${ }^{(8)}$. La mayoría de estudios $(75 \%)$ experimentaron con una muestra de 40 pacientes ${ }^{(8,10,11)}$. Dos estudios compararon el tiempo de intervención quirúrgica, siendo en ambos menor el tiempo para la técnica de frenectomía con el láser de Nd: YAG $(p<0.05)^{(8,9)}$. Un estudio encontró complicaciones posoperatorias como hemorragia (al utilizar el escalpelo) y exposición ósea al utilizar el láser de Nd:YAG ${ }^{(8)}$. Respecto a la evaluación del dolor posoperatorio tres estudios concuerdan en que este es menor con la técnica que aplicó el láser $(p<0.01)^{(9-11)}$. El estudio de Júnior y cols ${ }^{(8)}$ encontró mejores resultados con la técnica del escalpelo al tercer día de evaluación mas no en los otros días de evaluación. En los estudios de Kara(10) y Haytac $^{(11)}$ el dolor al masticar y el disconfort al hablar fueron menores con la técnica del láser $(p<0.01)$, en el estudio de Akpinar ${ }^{(9)}$ se encontró resultados similares pero no significativos $(p>0.05)$ (Tabla 1).

\section{DISCUSIÓN}

Frente a la presencia de un frenillo aberrante se plantea dos procedimientos para su tratamiento: frenectomía y frenotomía. La frenectomía implica la remoción completa del frenillo incluida su adherencia al hueso; mientras que la frenotomía la incisión y recolocación de la adherencia del frenillo(12,13).

La técnica de frenectomía con el uso de bisturíes fue planteada como 
Tabla 1. Ensayos clínicos que evaluaron los resultados clínicos de la remoción de frenillos aberrantes. VAS= Escala Visual Análoga.

\begin{tabular}{|c|c|c|c|c|}
\hline Estudio & $\begin{array}{l}\text { Tipo de } \\
\text { estudio }\end{array}$ & Procedimientos & Muestra & Resultados \\
\hline $\begin{array}{l}\text { Júnior y cols } \\
2013^{(8)}\end{array}$ & $\begin{array}{l}\text { Ensayo } \\
\text { clínico no } \\
\text { aleatorizado } \\
\text { multicéntrico }\end{array}$ & $\begin{array}{l}\text { Escalpelo } \\
\text { (Cirugía } \\
\text { convencional) } \\
\text { versus láser } \\
\text { Nd:YAG }\end{array}$ & $\begin{array}{l}\mathrm{N}=40 \\
\text { Edad=20,9 } \pm 10,3 \\
\text { Control: } \mathrm{n}=22 \\
\text { Experimental: } \mathrm{n}=18\end{array}$ & $\begin{array}{l}\text { - Tiempo de cirugía (min): } \\
\text { Escalpelo: 10,2 } \pm 1.8 \\
\text { Nd:YAG= 7,7 } \pm 1,9 \text { ( } p<0.001) \\
\text { - Dolor posoperatorio (VAS 0-10): } \\
\text { Menor dolor al tercer día en el grupo escalpelo. } \\
\text { - Dolor al hablar (VAS): } \\
\text { Menor dolor al tercer día en el grupo escalpelo. } \\
\text { - Disconfort al masticar (VAS): } \\
\text { Similar entre los grupos según los tiempos de evaluación. } \\
\text { - Complicaciones: } \\
\text { Tres pacientes (7,5\%). Una hemorragia con escalpelo y dos } \\
\text { exposiciones de hueso a nivel de la encía con el uso del láser. }\end{array}$ \\
\hline $\begin{array}{l}\text { Akpinar y cols } \\
2015^{(9)}\end{array}$ & $\begin{array}{l}\text { Ensayo } \\
\text { clínico } \\
\text { aleatorizado }\end{array}$ & $\begin{array}{l}\text { Escalpelo } \\
\text { (Cirugía } \\
\text { convencional) } \\
\text { versus láser } \\
\text { Nd:YAG }\end{array}$ & $\begin{array}{l}\mathrm{N}=99 \\
\text { Edad= 29,75 } \pm 11,58 \\
\text { Control: } \mathrm{n}=44 \\
\text { Experimental: } \mathrm{n}=45\end{array}$ & $\begin{array}{l}\text { - Tiempo de cirugía (min): } \\
\text { Escalpelo: 9,93 } \pm 3,32 \\
\text { Nd:YAG= 8,84 } \pm 3,11 \text { (p>0.05) } \\
\text { - Dolor posoperatorio (VAS 0-100): } \\
\text { Escalpelo: 0,2 (primer día), 0,1 (tercer día), } 0 \text { (sétimo día), } 0 \text { (décimo } \\
\text { día). } \\
\text { Nd: YAG: 0,1 (primer día), } 0 \text { (tercer día), } 0 \text { (sétimo día), } 0 \text { (décimo día). } \\
\text { - Dolor al hablar (VAS): } \\
\text { Escalpelo: 0,1 (primer día), } 0 \text { (tercer día), } 0 \text { (sétimo día), } 0 \text { (décimo día). } \\
\text { Nd: YAG: 0,1 (primer día), } 0 \text { (tercer día), } 0 \text { (sétimo día), } 0 \text { (décimo día). } \\
\text { - Disconfort al masticar (VAS): } \\
\text { Escalpelo: 0,1 (primer día), } 0 \text { (tercer día), } 0 \text { (sétimo día), } 0 \text { (décimo día). } \\
\text { Nd: YAG: } 0 \text { (primer día), } 0 \text { (tercer día), } 0 \text { (sétimo día), } 0 \text { (décimo día). } \\
\text { - Complicaciones: } \\
\text { Ninguna }\end{array}$ \\
\hline $\begin{array}{l}\text { Kara C } \\
2008^{(10)}\end{array}$ & $\begin{array}{l}\text { Ensayo } \\
\text { clínico } \\
\text { aleatorizado } \\
\text { cruzado }\end{array}$ & $\begin{array}{l}\text { Escalpelo } \\
\text { (Cirugía } \\
\text { convencional) } \\
\text { versus láser } \\
\text { Nd:YAG }\end{array}$ & $\begin{array}{l}\mathrm{N}=40 \\
\text { Edad=--- } \\
\text { Control: } \mathrm{n}=20 \\
\text { Experimental: } \mathrm{n}=20\end{array}$ & 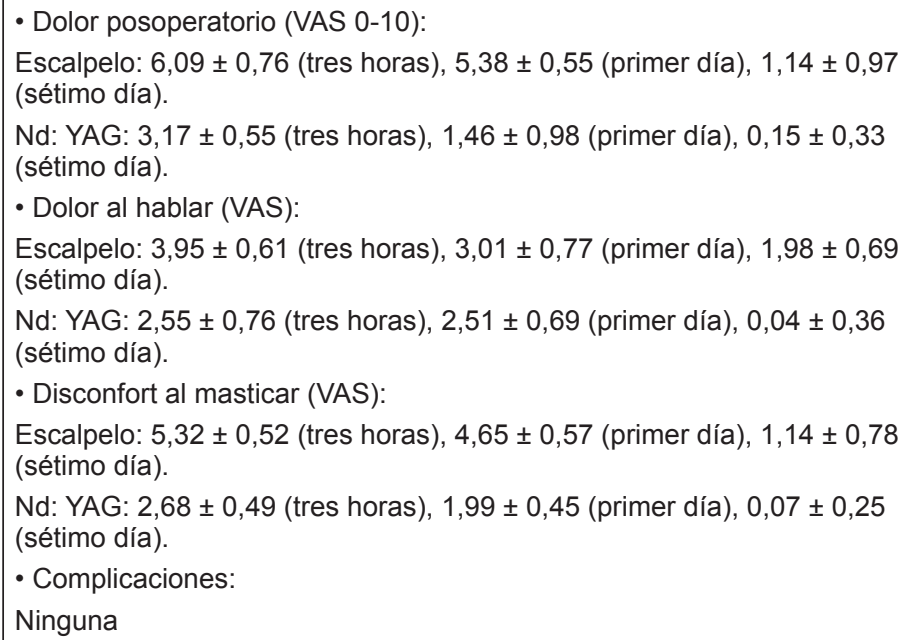 \\
\hline $\begin{array}{l}\text { Haytac y } \\
\text { Ozcelik } \\
2006^{(11)}\end{array}$ & $\begin{array}{l}\text { Ensayo } \\
\text { clínico } \\
\text { aleatorizado }\end{array}$ & $\begin{array}{l}\text { Escalpelo } \\
\text { (Cirugía } \\
\text { convencional) } \\
\text { versus láser } \mathrm{CO}_{2}\end{array}$ & $\begin{array}{l}\mathrm{N}=40 \\
\text { Edad= --- } \\
\text { Control: } \mathrm{n}=20 \\
\text { Experimental: } \mathrm{n}=20\end{array}$ & 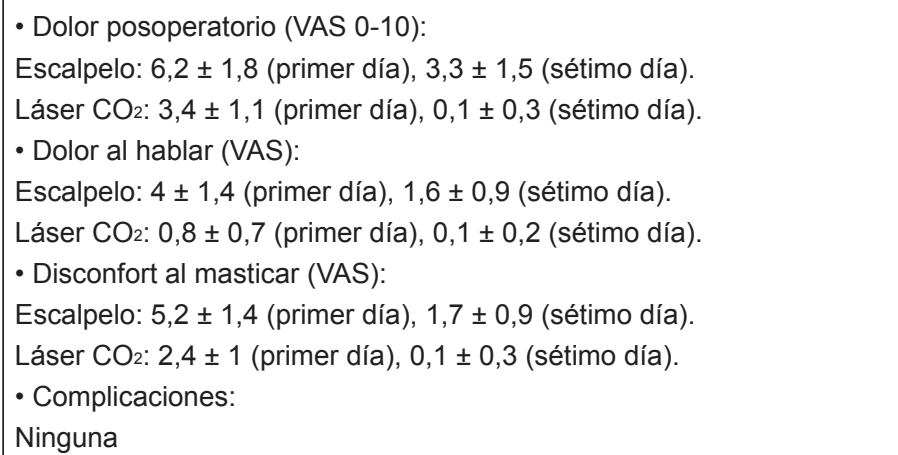 \\
\hline
\end{tabular}


la técnica convencional y clásica para remover a los frenillos aberrantes; sin embargo, esta técnica posee las desventajas de ocasionar mayor sangrado y mayor posibilidad de complicaciones posoperatorias. Por el contrario, las técnicas que utilizan el láser reducen el riesgo de sangrado y complicaciones pero pueden ocasionar quemaduras, riesgo de explosión al usar gases, riesgo de interferir con marcapasos y producir humo quirúrgico(13).

Las ventajas y desventajas clínicas de cada láser han sido reportadas principalmente en forma de reportes clínicos, existiendo escasos estudios que comparen los resultados posoperatorios entre las distintas técnicas. Según los ensayos clínicos encontrados, las comparaciones se pueden realizar entre las técnicas convencionales con el escalpelo y las técnicas que utilizan el láser $\mathrm{Nd}$ : YAG y el láser $\mathrm{CO}_{2}$ siendo las principales variables a contrastar el tiempo de intervención quirúrgica, el dolor posoperatorio al hablar y masticar, y las complicaciones posquirúrgicas.

Las técnicas con láser no necesitan anestesia, generan menos dolor, otorgan mejor visibilidad al operar, no necesitan apósitos periodontales, ofrecen mejor cicatrización y una menor cicatriz ${ }^{(14)}$. El láser permite esterilizar la zona y su uso no requiere el uso de suturas ${ }^{(15)}$. De los estudios analizados, uno reportó complicaciones posquirúrgicas relacionadas con la exposición de hueso a nivel de la encía adherida luego de una semana de intervención ${ }^{(8)}$. Los autores plantean que la inatención del operador al utilizar el láser y generar mayor energía térmica pudo explicar dichas complicaciones ${ }^{(8)}$.

El láser de $\mathrm{Nd}$ : YAG y el láser $\mathrm{CO}_{2}$ evidenciaron mejores resultados respecto al dolor posoperatorio y disconfort al hablar y masticar ${ }^{(9-11)}$. Se ha utilizado el láser de $\mathrm{CO}_{2}$ para frenectomías linguales, con ventajas de ser simple, rápida, ocasionar poco dolor, ausencia de infecciones, ausencia de parestesias además de dejar poca o nada de cicatrices ${ }^{(16)}$. El estudio de Haytac y cols ${ }^{(11)}$ comparó el grado de dolor posoperatorio al realizar frenectomías con bisturí y con láser de $\mathrm{CO}_{2}$, el grupo láser tuvo menos dolor y complicaciones funcionales (habla e hinchazón) $(p<0,001)$, además de requerir menos analgésicos $(p<0,001)$. Concluyen que el láser de $\mathrm{CO}_{2}$ ofrece una alternativa segura, efectiva y aceptable para la realización de frenectomías.

Señalar además que los tejidos orales (principalmente los tejidos de revestimiento) poseen más de $90 \%$ de agua dentro de su composición y considerando la afinidad del diodo por tejidos húmedos, el láser diodo está muy indicado para cirugías orales ${ }^{(17)}$.
Si bien los ensayos analizados evidencian mejores resultados respecto a la percepción del dolor con las técnicas que utilizan el láser, la superioridad de las técnicas con el láser versus las técnicas convencionales aún no ha sido demostrada ${ }^{(2)}$. Existieron limitantes durante la selección y análisis de los artículos para la presente revisión sistemática; la principal radicó en la escaza bibliografía existente respecto a técnicas quirúrgicas comparables y una abundancia de reportes clínicos sobre la remoción de los frenillos aberrantes. La escasa cantidad de ensayos clínicos relacionados con la temática limita un mayor análisis de las ventajas y desventajas de las técnicas quirúrgicas sobre frenectomía y frenotomía. Otra limitante durante el análisis fueron las escalas utilizadas durante la evaluación posquirúrgica (Escala Visual Análoga), escala que valora la percepción de un paciente y cuyos resultados son subjetivos y no permiten valorar un análisis más preciso de los resultados. Es necesario recomendar mayores ensayos clínicos que comparen técnicas quirúrgicas para la remoción de frenillos aberrantes así como la utilización de variables y/o escalas objetivas (exposición de sonrisa, complicaciones, longitud del labio, etc.) para valorar los resultados posoperatorios.

\section{CONFLICTO DE INTERÉS}

Los autores no mostramos ningún tipo de conflicto de interés con respecto al artículo.

\section{FUENTE DE FINANCIAMIENTO}

La presente investigación no ha sido financiada por alguna institución.

\section{RELEVANCIA CLÍNICA}

La inserción de los frenillos raramente ocasiona patologías dentro de la cavidad bucal; sin embargo, cuando lo hace puede ocasionar problemas periodontales, fonéticos, ortodónticos y protésicos. La presente revisión resume los principales resultados clínicos que se logran al remover los frenillos aberrantes a través de las técnicas de frenectomía y frenotomía. Los resultados facilitarán la toma de decisiones para la práctica clínica según la evidencia científica actualizada.

\section{Bibliografía}

1. Grados SP, Castro YR, Bravo FC. Consideraciones clínicas en el tratamiento quirúrgico periodontal. 1a. edición. Caracas: AMOLCA; 2014.

2. Delli K, Livas C, Sculean A, Katsaros C, Bornstein M. Facts and myths regarding the maxillary midline frenum and its treatment: A systematic review of the literature. Quintessence Int. 2013;44:177-87.

3. Ward VJ. A clinical assessment of the use of the free gingival graft for correcting localized recession associated with frenal pull. J Periodontol. 1974;45(2):78-83. 4. Devishree SK. Frenectomy: A review with the reports of surgical techniques. J Clin Diagn Res. 2012;6(9):1587-92.

5. Prabhuji ML, Madhu SS, Armeya GM. Frenectomy review comparison of conventional techniques with diode laser. Laser. 2010;3:14-7.

6. Higgins JPT, Green S. Cochrane handbook for systematic reviews of interventions, Version 5.1.0 (updated March 2011). The Cochrane Collaboration, 2011. [Cited 16/11/2016]. Disponible en: http://www.cochrane-handbook.org.

7. Schulz KF, Altman DG, Moher D, CONSORT Group. CONSORT 2010 statement Updated guidelines for reporting parallel group randomized trials. Ann Intern Med. 2010;152:726-32.

8. Júnior RM, Gueiros LA, Silva IH, de Albuquerque Carvalho A, Leão JC. Labia frenectomy with $\mathrm{Nd}$ :YAG laser and conventional surgery: a comparative study. Lasers Med Sci. 2015;30(2):851-6.

9. Akpinar A, Toker H, Lektemur Alpan A, Calisir M. Postoperative discomfort after $\mathrm{Nd}$ : YAG laser and conventional frenectomy: comparison of both genders. Aust Dent J. 2015;16;61(1):71-75
10. Kara C. Evaluation of patient perceptions of frenectomy: a comparison of $\mathrm{Nd}$ :YAG laser and conventional techniques. Photomed Laser Surg. 2008;26(2):147-52.

11. Haytac MC, Ozcelik O. Evaluation of patient perceptions after frenectomy operations: a comparison of carbon dioxide laser and scalpel techniques. J Periodontol. 2006;77(11):1815-9.

12. Pié-Sánchez J, España-Tost AJ, Arnabat-Domínguez J, Gay-Escoda C Comparative study of upper lip frenectomy with the CO2 laser versus the Er, Cr:YSGG laser. Med Oral Patol Oral Cir Bucal. 2012;17(2):e228-32.

13. Kato J, Wijeyeweera RL. The effect of $\mathrm{CO} 2$ laser irradiation on oral soft tissue problems in children in Sri Lanka. Photomed Laser Surg. 2007;25(4):264-8.

14. Hungund $S$, Dodani K, Kambalyal $P$, Kambalyal $P$.Comparative results of frenectomy by three surgical techniques- conventional, unilateral displaced pedicle flap and bilateral displaced pedicle flap. Dentistry. 2013;4(1):2-6.

15. Gamez RJ. Frenectomía convencional frente a frenectomía láser. Odont Act. 2008;5(60):26-32.

16. Chiapasco M. Tácticas y técnicas en cirugía oral. Caracas. Amolca. 2010

17. Kaur P, Paul YD, Kaushal S, Bhatia A, Vaid R, Sharma R. Management of the upper labial frenum: a comparison of conventional surgical and lasers on the basis of visual analogue scale on patients perception. J Periodontal Med Clin Pract 2014;1(1):38-44 